Please do not remove this page

RMIT

UNIVERSITY

\title{
Investigation of resonantly enhanced modulators on LiNbO3 using FEM and numerical optimization technique
}

Nguyen, Giang; Mitchell, Arnan; Visagathilagar, Yuvaraja

https://researchrepository.rmit.edu.au/esploro/outputs/9921858011001341/filesAndLinks?institution=61RMIT_INST\&index=null

Nguyen, G., Mitchell, A., \& Visagathilagar, Y. (2004). Investigation of resonantly enhanced modulators on LiNbO3 using FEM and numerical optimization technique. IEEE Journal of Lightwave Technology, 22, 526-533. https://doi.org/10.1109/JLT.2003.821746

Published Version: https://doi.org/10.1109/JLT.2003.821746

Repository homepage: https://researchrepository.rmit.edu.au

(c) 2004 IEEE. Personal use of this material is permitted. However, permission to reprint/republish this material for advertising or promotional purposes or for creating new collective works for resale or redistribution to servers or lists, or to reuse any copyrighted component of this work in other works must be obtained from the IEEE.

Downloaded On 2023/04/26 20:34:41 +1000 


\title{
Investigation of Resonantly Enhanced Modulators on $\mathrm{LiNbO}_{3}$ Using FEM and Numerical Optimization Technique
}

\author{
Thach Giang Nguyen, Arnan Mitchell, Member, IEEE, and Yuvaraja S. Visagathilagar, Member, IEEE
}

\begin{abstract}
Highly efficient resonantly enhanced modulators on $X$-cut $\mathrm{LiNbO}_{3}$ are investigated through the use of numerical optimization. We describe the optimization technique and present a new design trend relating the link-gain efficiency of optical radio systems that employ external modulation to the choice of modulator electrode geometric dimensions, especially the electrode gap. Using this optimization technique, resonant-type modulators with link gain enhancement up to $6 \mathrm{~dB}$ are achievable while maintaining excellent return loss at a resonant frequency of $1.8 \mathrm{GHz}$. The characteristics of both the optical waveguide and the coplanar electrode are characterized by finite-element simulation.
\end{abstract}

Index Terms-Adaptive simulated annealing $\mathrm{LiNbO}_{3}$, link gain, narrow bandwidth, optical modulator, optimization, resonant enhancement.

\section{INTRODUCTION}

$\mathbf{M}$ ACH-ZEHNDER modulators (MZMs) on $\mathrm{LiNbO}_{3}$ have proven to be essential devices for optical fiber communication systems. Conventional modulators commonly employ a traveling-wave electrode structure [1]. This device offers large bandwidth but only modest modulation efficiency, which leads to high half-wave voltage $\left(V_{\pi}\right)$. $\mathrm{LiNbO}_{3}$ modulators with bandwidths up to $100 \mathrm{GHz}$ have been demonstrated [2]. However, the radio-frequency (RF) links that use MZMs suffer substantial RF link loss due to high $V_{\pi}$. In some telecommunication applications, such as in radio-on-fiber [3], only a narrow bandwidth around the center frequency is required, but these applications possess stringent RF link-gain requirements for practical use [4]. Thus, it is desirable to reduce the modulator bandwidth around the center frequencies to achieve highly efficient modulator devices using resonant enhancement techniques.

Recently, several resonant-type modulator structures have been proposed in the literature [5]-[9]. These modulators provide very strong modulation efficiency per unit length and low half-wave voltage length product $(25 \mathrm{Vmm}$ in [9], for example). However, these devices focused only on modulation efficiency of very short electrode structures (on the order of millimeters) for the active section. The consequent result is that the actual modulation efficiency is low compared with standard traveling modulators with large electrode geometries [2] and thus may not offer significant benefit in terms of RF link gain efficiency. In [10], the electrode structure was optimized so that

Manuscript received June 11, 2003; revised August 18, 2003.

The authors are with the School of Electrical and Computer Engineering,

Royal Melbourne Institute of Technology, 3001 Melbourne, Australia.

Digital Object Identifier 10.1109/JLT.2003.821746 electrode length was made large. By lengthening the electrode, the $V_{\pi}$ can be reduced significantly, from $17 \mathrm{~V}$ in [9] to $5.8 \mathrm{~V}$ in [10] with a 29.624-mm electrode. However, that $V_{\pi}$ is still higher than the $V_{\pi}$ of standard traveling modulators [2]. Hence, good modulation enhancement does not always lead to good link-gain enhancement.

In another approach [11], a very long traveling-wave electrode was employed to reduce the drive voltage. In order to attain a long coplanar waveguide (CPW) electrode, a large gap was used to reduce electrode loss. With a very long electrode, the drive voltage was lowered significantly to less than $1.2 \mathrm{~V}$ with dual drive.

In this paper, we theoretically investigate a Fabry-Pérot-type resonantly enhanced modulator [12] on $X$-cut $\mathrm{LiNbO}_{3}$ with long electrodes. Particular emphasis is placed on the tradeoff between improving inherent $V_{\pi}$ with weak enhancement and reducing loss at the expense of the inherent $V_{\pi}$ in order to get strong modulation enhancement.

The theoretical analysis is performed using numerical optimization to explore highly efficient resonantly enhanced modulators. It will be shown that by optimizing electrode geometric parameters such as the electrode gap, electrode width, electrode thickness, and buffer-layer thickness, it is possible to obtain a strong modulation enhancement while at the same time reducing the inherent $V_{\pi}$. Thus, the total net effect is an improved link-gain performance.

In Section II, the theory of operation and the configuration of the resonantly enhanced MZM (RE-MZM) is reviewed, and an expression for the modulation efficiency as a function of half-wave voltage is also developed. The finite-element method (FEM) simulation techniques used to predict RF performance and inherent $V_{\pi}$ are outlined in Section III. A brief parameter study of the impact of electrode geometry on RF attenuation and inherent $V_{\pi}$ is presented in Section IV. Section V presents the optimization approach used in this investigation. The results of the optimized RE-MZM based on the applied optimization scheme are presented in Section VI. Section VII presents a summary and suggestions for future work.

\section{ResonAntLy ENHANCED Modulator AND RF LinK GAin}

As presented in [12], introducing load mismatches at both input and output ports of the modulator to form a Fabry-Pérot-type cavity will result in most of the microwave signal's forming resonant standing waves. Strong field enhancement at the resonant frequency can be obtained, provided 


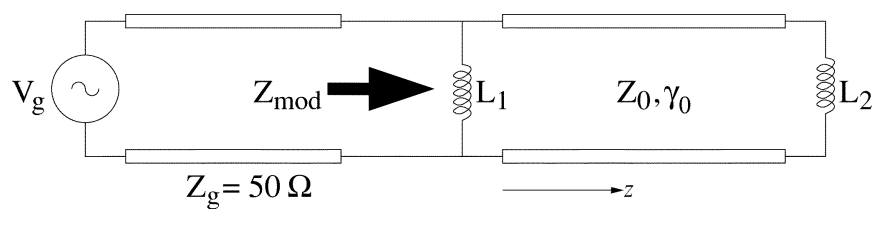

Fig. 1. Equivalent circuit of resonantly enhanced modulator.

low-loss electrodes can be achieved. With terminations at both input and output ports, good return loss is also achievable. The load mismatches can be realized by shorted CPW stub circuits [12], [13], which are effectively inductively coupled. In this paper, the terminations are simply modeled as inductors. The equivalent circuit model of a resonant electrode structure is shown in Fig. 1.

The link gain of an RF-over-fiber link that employs an external modulation scheme when the modulator is biased at quadrature and under small signal operation is expressed as [14]

$$
\rho_{G}(f)=2 R_{L}\left(\frac{\pi r P_{o} \alpha_{m} \alpha_{o p t}}{2} \frac{R_{0}}{R_{0}+R_{L}}\right)^{2} M_{G}(f) .
$$

The link gain factor of the modulator is given by

$$
M_{G}(f)=\frac{\left(1-|\Gamma|^{2}\right)}{\operatorname{Re}\left\{\frac{1}{Z_{\bmod }}\right\} V_{\pi}^{2}(f)}
$$

where $\Gamma=\left(Z_{\text {mod }}-Z_{g}\right) /\left(Z_{\text {mod }}+Z_{g}\right)$ is the voltage reflection coefficient at modulator input, $Z_{\text {mod }}$ is the total impedance of the modulator, which includes the electrode characteristic impedance and the terminations, $Z_{g}$ is the impedance of the microwave source, and other variables are as in [14]. $M_{G}$ is a good indication of how resonantly enhanced modulators improve the link gain as it includes the effects of modulation efficiency, half-wave voltage, and impedance matching.

The frequency-dependent half-wave voltage is defined as

$$
V_{\pi}(f)=\frac{\pi V_{\text {in }}}{\Delta \Phi}
$$

where $V_{\text {in }}$ is the input voltage amplitude and $\Delta \Phi$ is the total accumulated phase change in two arms of a modulator due to Pockel's effect. $\Delta \Phi$ is given by

$$
\Delta \Phi=\frac{\pi}{V_{\pi}} \Delta \varphi V_{\text {in }}
$$

where $V_{\pi}$ defines the inherent strength of electrooptic interaction of the electrode structure. The frequency-dependent normalized induced phase shift $\Delta \varphi$ is given by

$$
\Delta \varphi=\frac{1}{V_{\text {in }}} \int_{0}^{l} V(z) e^{-j(2 \pi f / c) n_{o} z} d z
$$

where $n_{o}$ is the effective index of the guided optical mode, $V(z)$ is the complex voltage at point $z$ along the active section of electrode, and $l$ is the length of the active section of the electrode.

Inherent $V_{\pi}$ is a property of the modulator cross section. $\Delta \varphi$ is related to electrode loss, index match, and resonant enhancement. $\Delta \varphi$ shows the modulation efficiency of the resonant electrode.

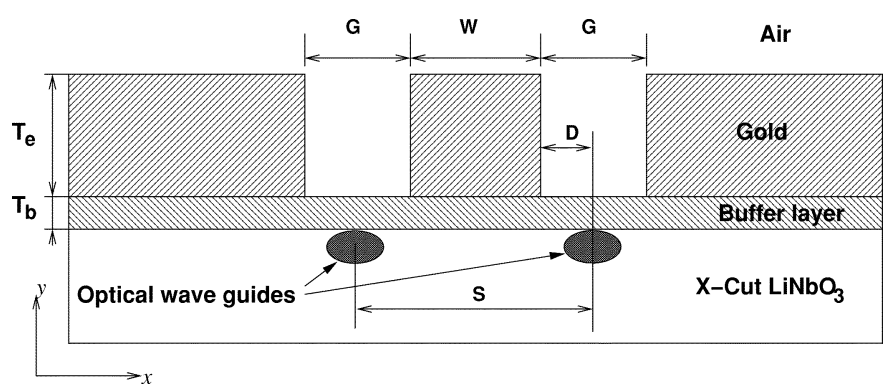

Fig. 2. Cross section of the modulator

As can be seen from (1)-(4), the link gain is dependent on inherent $V_{\pi}$, modulation efficiency $\Delta \varphi$, and total modulator impedance $Z_{\text {mod }}$. Optimal modulator impedance at the resonant frequency can be obtained by carefully choosing the terminations. Hence, electrode characteristic impedance is not of primary importance. Furthermore, the electrode microwave index is not highly significant for narrow-band operation; thus, some of the design tradeoffs usually associated with broad-band modulator design can be relaxed.

For resonant-type modulators, modulation efficiency is mainly determined by electrode loss [9], [12]. In [5] and [9], the length of the electrode is shortened to reduce total electrode loss. If we can reduce electrode loss so that the electrode can be made longer to get longer interaction length, the modulation efficiency will be higher. Therefore, it is desirable to design electrode structures that simultaneously provide low inherent $V_{\pi}$ and low loss. In the next sections, the dependency of electrode loss and inherent $V_{\pi}$ on electrode structure is explored through the use of the FEM.

\section{FEM SIMULATION TECHNIQUES}

Fig. 2 shows the cross section of a Mach-Zehnder optical intensity modulator with CPW electrode on $X$-cut $\mathrm{LiNbO}_{3}$. W is the width of the hot electrode, $G$ is the gap between the hot electrode and the ground plane, $T_{b}$ is the thickness of the buffer layer, $T_{e}$ is the electrode thickness, $S$ is the distance between two optical waveguides, and $D$ is the distance from the center of the first optical waveguide to the hot electrode wall.

\section{A. Simulation of Microwave Properties}

The microwave properties of the CPW have been simulated using a two-dimensional (2-D) vector finite-element electromagnetic simulator. This implementation uses a first-order edge element as described in [15]. A subspace iteration eigenvalue solver is used to identify the eigenvalues and eigenvectors of the coplanar structure. From these eigensolutions, the characteristics, including microwave effective index $\left(N_{m}\right)$, microwave propagation loss $\left(\alpha_{m}\right)$, and characteristic impedance $\left(Z_{0}\right)$, can be calculated. These characteristics describe the microwave behavior of the CPW cross section.

\section{B. Simulation of Inherent $V_{\pi}$}

Inherent $V_{\pi}$ can be calculated as [16]

$$
V_{\pi}=\frac{\lambda}{n_{e}^{3} r_{33} \sigma}
$$




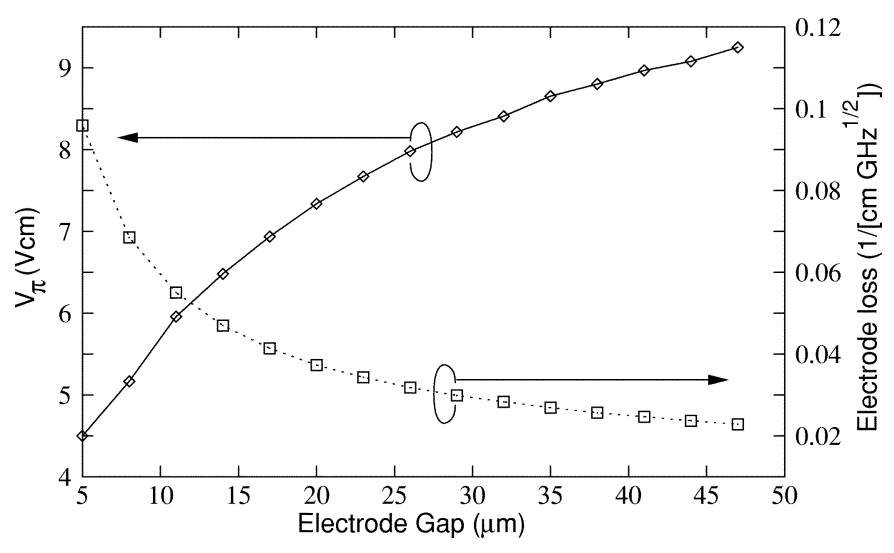

Fig. 3. Variations of electrode loss and inherent $V_{\pi}$ with electrode gap $G$.

where $\lambda$ is the free-space optical wavelength, $n_{e}$ is the extraordinary refractive index of the substrate, $r_{33}$ is the pertinent electrooptic coefficient, and $\sigma$ is the overlap factor between the electric field of the lightwave and the electric field induced by the electrode. Here we consider the push-pull configuration; therefore, the total overlap factor $\sigma$ is equal to the sum $\left|\sigma_{1}\right|+\left|\sigma_{2}\right|$, where $\sigma_{1}$ and $\sigma_{2}$ are overlap factors for each one of the optical waveguides. The overlap factor of each waveguide is defined as

$$
\sigma_{i}=\frac{1}{V} \frac{\iint E_{\mathrm{op}_{i}}^{2} E_{\mathrm{el}} d x d y}{\iint E_{\mathrm{op}_{i}}^{2} d x d y}
$$

where $V$ is the applied voltage, $E_{\text {op }}$ is the normalized optical modal electric field, and $E_{\mathrm{el}}$ is the normalized electric field of the electrode structure. The optical field profile $E_{\text {op }}$ is calculated using a scalar, 2-D finite-difference simulation. The electric field $E_{\mathrm{el}}$ is approximated by the static electric field. This static electric field is calculated using a modification of the finite-element code of Section III-A that solves the diffusion equations for the static voltage.

\section{Electrode Structure Dependency of Electrode LOSS AND INHERENT $V_{\pi}$}

As stated previously, both the electrode loss and inherent $V_{\pi}$ should be reduced to increase the link gain. Using the FEM described in Section III, we now explore how these two parameters are affected by electrode cross-sectional dimensions. In all FEM calculations, the conductivity of gold is taken as $40.8 \times 10^{6} \mathrm{~s} / \mathrm{m}$. The optical waveguide width and thickness are $7 \mu \mathrm{m}$ and $1000 \AA$, respectively. The diffusion lengths of the optical waveguides are $4 \mu \mathrm{m}$. The modulators are designed to operate at $1.55-\mu \mathrm{m}$ wavelength.

\section{A. Electrode Gap}

Among the electrode cross-sectional parameters, the gap between hot electrode and the ground plane $G$ has a strong effect on both electrode loss and inherent $V_{\pi}$ [11]. Fig. 3 shows the relationship between electrode loss and inherent $V_{\pi}$ with variation in the electrode gap. In this case, the buffer layer thickness $T_{b}=0.2 \mu \mathrm{m}$, the electrode thickness $T_{e}=30 \mu \mathrm{m}$, and the hot-electrode width $W=16 \mu \mathrm{m}$. It can be noted that electrode loss reduces significantly with increasing electrode gap. However, the electrode gap should be reduced to lower inherent $V_{\pi}$.

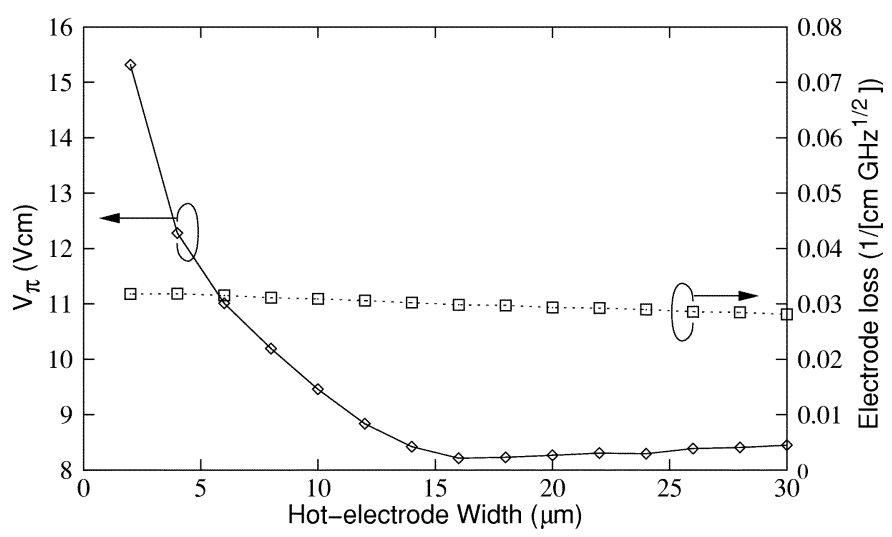

Fig. 4. Hot-electrode-width dependency of inherent $V_{\pi}$ and electrode loss. Here buffer layer thickness is $0.2 \mu \mathrm{m}$, electrode thickness is $30 \mu \mathrm{m}$, and the electrode gap is $29 \mu \mathrm{m}$. Minimum optical waveguide separation is $20 \mu \mathrm{m}$.

Therefore, it is not obvious whether a large or small gap should be used to optimize link gain. The tradeoff needs to be explored.

\section{B. Hot-Electrode Width}

For $X$-cut devices, two optical waveguides have to be placed in the gap between the hot electrode and ground plane to utilize the largest electrooptic coefficient $r_{33}$ of $\mathrm{LiNbO}_{3}$ as shown in Fig. 2. The optical waveguides should be placed at the points where they will get strongest electrooptic interaction to increase the overlap integral factor $\sigma$. Therefore, inherent $V_{\pi}$ is also determined by the optical waveguide positions. From FEM simulation, it is found that $D=2 \mu \mathrm{m}$ results in the strongest electrooptic interaction. This result agrees reasonably well with that reported in [17].

To ensure minimal coupling between optical waveguides to maintain good extinction ratio, the optical waveguides cannot be placed too close to each other. A minimum separation $(S \sim$ $20 \mu \mathrm{m}$ ) has to be maintained between two optical waveguides. Due to the restriction on waveguide separation, it not always possible to place both optical waveguides at optimal positions. Therefore, for the modulator fabricated on $X$-cut $\mathrm{LiNbO}_{3}$, inherent $V_{\pi}$ is strongly affected by the width of hot electrode $W$, as illustrated in Fig. 4. When the hot electrode is very narrow, two optical waveguides with minimum separation $S=20 \mu \mathrm{m}$ cannot be placed at optimal positions; thus, inherent $V_{\pi}$ is high. Once the electrode width is increased to above $16 \mu \mathrm{m}$, it is possible to place both electrodes in their optimal positions, as indicated by the flattening in $V_{\pi}$ in Fig. 4 . Fig. 4 also shows the variation of the electrode loss with hot-electrode width. It can be seen that the loss decreases only marginally as hot-electrode width is increased. Thus, a large electrode width should be used for optimal link gain.

\section{Buffer-Layer Thickness}

The buffer-layer thickness $T_{b}$ also plays a very important role in the design of the optical modulator. The variation of inherent $V_{\pi}$ with the buffer-layer thickness $T_{b}$ is shown in Fig. 5. It can be observed that the reduction in buffer-layer thickness leads to a significant decrease of inherent $V_{\pi}$. Hence, for the purpose of lowering inherent $V_{\pi}$, the buffer-layer should be made very thin. A finite buffer layer $\left(T_{b} \sim 0.2 \mu \mathrm{m}\right)$ is still required to minimize 


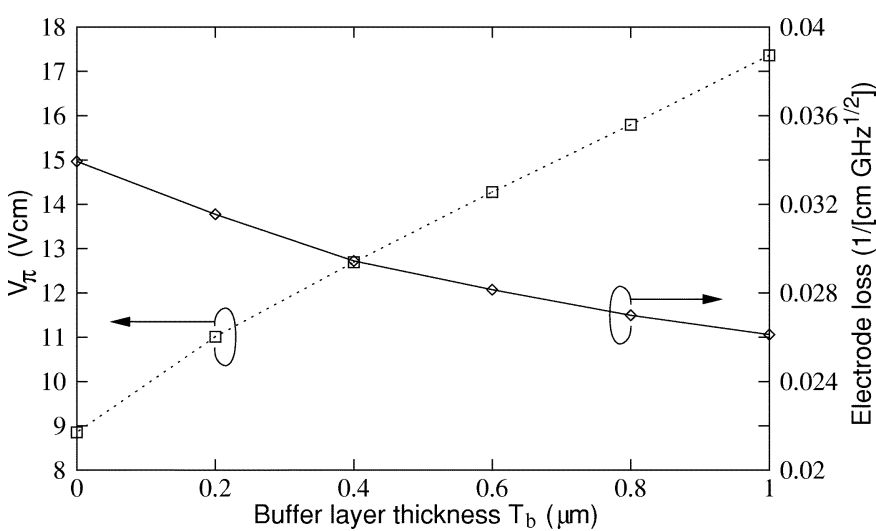

Fig. 5. Variations of inherent $V_{\pi}$ and electrode loss with the buffer-layer thickness $T_{b}$ of a CPW electrode with gap-width-gap configuration of 29-6-29 $\mu \mathrm{m}$ and electrode thickness of $30 \mu \mathrm{m}$.

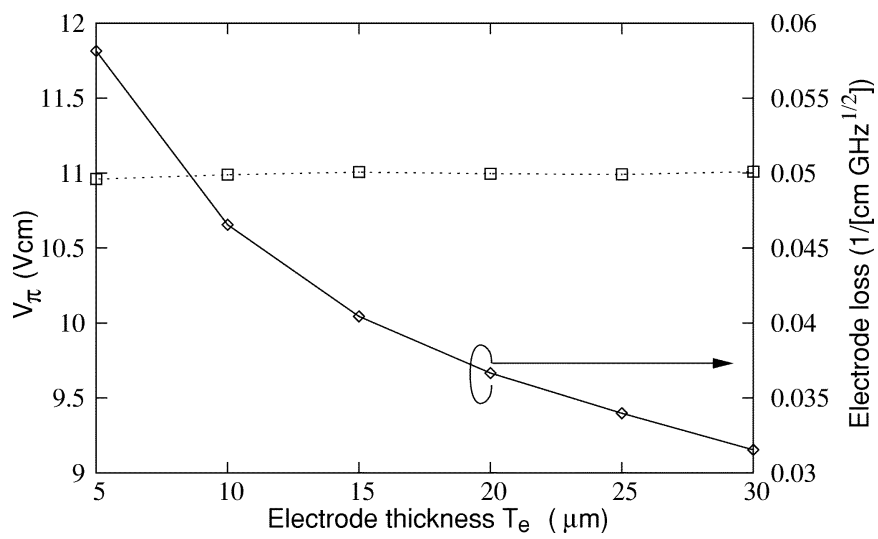

Fig. 6. Variations of electrode loss and inherent $V_{\pi}$ with the electrode thickness $T_{e}$. Here buffer-layer thickness $T_{b}$, electrode gap $G$, and electrode width $W$ were taken as $0.2,29$, and $6 \mu \mathrm{m}$, respectively.

optical loss [1]. The electrode structure with a thin buffer layer has a large microwave effective index, which increases velocity mismatch. Large velocity mismatch will impact modulation, but it is not necessarily detrimental for resonantly enhanced modulators. Also shown in Fig. 5 is the electrode loss as a function of buffer-layer thickness. The buffer-layer thickness has a weak effect on the electrode loss. The loss increases slightly as the buffer-layer thickness is reduced through the higher microwave effective index, so a thin buffer layer allows for lower inherent $V_{\pi}$ at the price of slightly increasing electrode loss.

\section{Electrode Thickness}

The last electrode dimension to be considered is the electrode thickness $T_{e}$. Its effects on electrode loss and inherent $V_{\pi}$ are shown in Fig. 6. It can be noted that electrode loss decreases significantly with the increase of electrode thickness $T_{e}$. Inherent $V_{\pi}$ is almost independent of electrode thickness. Thus, the electrode thickness can be easily optimized for low electrode loss with almost no penalty in inherent $V_{\pi}$.

\section{E. Summary}

To summarize, inherent $V_{\pi}$ depends strongly on electrode gap, width, and buffer-layer thickness, whereas electrode loss is mainly determined by electrode gap and electrode thickness and

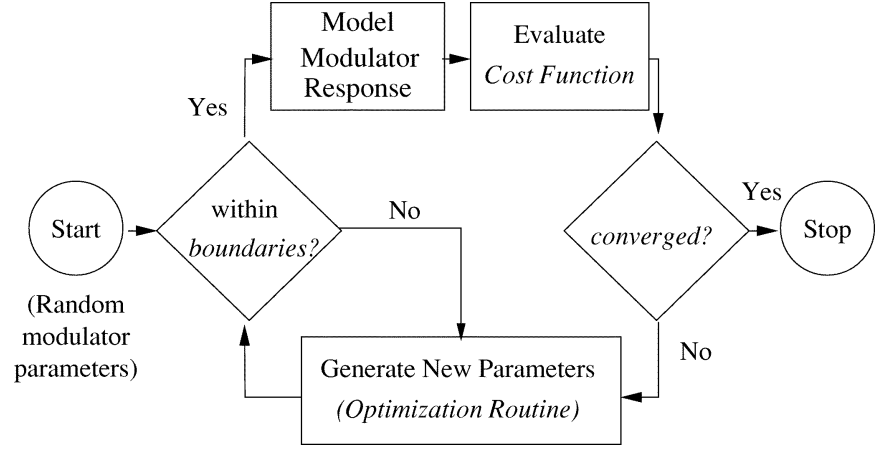

Fig. 7. Flowchart of the automatic synthesis procedure.

is only slightly dependent on buffer-layer thickness. Electrode thickness and electrode width can be optimized to achieve low electrode loss and low inherent $V_{\pi}$ without any conflict. However, the goals of simultaneously lowering of both inherent $V_{\pi}$ and electrode loss compete when considering buffer-layer thickness and, especially, electrode gap. A tradeoff must be made. In the following sections, a numerical optimization technique will be employed to explore this tradeoff.

\section{OPTIMIZATION APPROACH}

\section{A. Optimization Procedure}

Fig. 7 presents a diagram of the optimization procedure used in this investigation. This is based on a similar procedure used in [18]. It begins with a random set of modulator parameters. These are then checked to see if they fall within parameter boundaries. If the parameters are within boundaries, the modulator optical response is modeled, and the results are assessed using a cost function; otherwise, a new set of parameters has to be generated. The result of the current cost function is compared with that of the previous iteration to determine if the solutions have converged. If the normalized change in cost is smaller than a specified tolerance, then the solution is deemed converged, and the optimization is halted; otherwise, the current parameters and cost are passed to the optimization routine, where a new set of parameters is generated. This new set of parameters is checked against the parameter boundaries, and the cycle continues until the convergence criterion is met.

Adaptive simulated annealing (ASA) [19] is used as the optimization routine. ASA is an efficient search strategy and is statistically guaranteed to find function optima [20].

\section{B. Optimization Parameters and Parameter Boundaries}

The optimization routine seeks the value of electrode crosssectional parameters (electrode thickness $T_{e}$, buffer-layer thickness $T_{b}$, width $W$, and gap $G$ ), electrode length $l$, and terminations $L_{1}, L_{2}$ to increase the link gain. Loose constraints are applied to optimization variables to ensure the optimized modulator can be practically realized. The optimization routine only searches for variable values within their boundaries.

With current processing technology, the electrode and buffer layer can be fabricated with thickness up to 30 and $1 \mu \mathrm{m}$, respectively. The length of the electrode must be less than $6 \mathrm{~cm}$ [11]. A bias voltage can be applied to the same electrode using 
a bias-T device. A maximum value of $6 \mathrm{nH}$ is placed on the terminations so that they can be realized with shorted CPW stubs. Parameter boundaries are summarized as

$$
\begin{aligned}
5 \mu \mathrm{m} & \leq T_{e} \leq 30 \mu \mathrm{m} \\
0.2 \mu \mathrm{m} & \leq T_{b} \leq 1 \mu \mathrm{m} \\
4 \mu \mathrm{m} & \leq W \leq 30 \mu \mathrm{m} \\
5 \mu \mathrm{m} & \leq G \leq 47 \mu \mathrm{m} \\
1 \mathrm{~cm} & \leq l \leq 6 \mathrm{~cm} \\
0 & \leq L_{1}, \quad L_{2} \leq 6 \mathrm{nH} .
\end{aligned}
$$

\section{Modulator Response Model}

A numerical network model [21] was used to simulate the electrooptic response of the modulator. The network model uses the microwave properties of the electrode (electrode loss, microwave index, and characteristic impedance) and inherent $V_{\pi}$ to model the electrooptic response. Microwave properties and the inherent $V_{\pi}$ of modulators with a set of different geometrical structures were calculated in advance by FEM methods, as presented in Section III and stored in a table. Whenever the optimization routine generates a new set of modulator geometric parameters, it will look up the data table to find the coresponding microwave properties and inherent $V_{\pi}$. The data are then forwarded to the network model program to find the electrooptic response of the modulator. Since the FEM calculation was not called for each iteration, only modest optimization times were required.

\section{Optimization Cost Function}

The primary objective of the optimization is to find a modulator configuration that offers as high as possible link gain but maintains acceptable RF return loss of $-15 \mathrm{~dB}$. The optimization thus attempts to minimize the link loss, which is the reciprocal of the link gain. If the return loss is greater than the $-15-\mathrm{dB}$ constraint, a significant penalty is applied to the cost function. Optimization is conducted at a single resonant frequency. Mathematically, the cost function can be written as

$$
\text { Cost }= \begin{cases}\frac{\operatorname{Re}\left\{\frac{1}{Z_{\bmod }}\right\} V_{\pi}^{2}(f)}{\left(1-|\Gamma|^{2}\right)}, & \text { if } S_{11}<-15 \mathrm{~dB} \\ 10^{10}, & \text { else. }\end{cases}
$$

\section{OPTIMIZATION RESULTS}

Having developed a suitable optimization algorithm in Section $\mathrm{V}$, we now employ it to explore highly efficient resonantly enhanced modulators. In all optimization cases, a typical value $n_{o}=2.15$ is assumed for the effective index of optical waveguides. Frequency $f=1.8 \mathrm{GHz}$ is chosen for investigation since it is widely used in current telecommunications such as in a Global System for Mobile Communications (GSM) architecture.

\section{A. Optimization With Buffer-Layer Thickness}

In the first optimization case, the tradeoff between inherent $V_{\pi}$ and the electrode loss associated with buffer-layer thickness was examined. In this case, the electrode gap and width were
TABLE I

OPTIMIZATION RESULTS: CASE 1

\begin{tabular}{l|l}
\hline Parameter & Value \\
\hline \hline Electrode gap $(G)$ & $29 \mu \mathrm{m}$ \\
\hline Electrode width $(W)$ & $6 \mu \mathrm{m}$ \\
\hline Buffer layer thickness $\left(T_{b}\right)$ & $0.2 \mu \mathrm{m}$ \\
\hline Electrode thickness $\left(T_{e}\right)$ & $30 \mu \mathrm{m}$ \\
\hline Electrode length $(l)$ & $5.335 \mathrm{~cm}$ \\
\hline Input termination $\left(L_{1}\right)$ & $2.06332 \mathrm{nH}$ \\
\hline Output termination $\left(L_{2}\right)$ & $1.96548 \mathrm{nH}$ \\
\hline Microwave index $\left(N_{m}\right)$ & 2.59484 \\
\hline Electrode impedance $\left(Z_{0}\right)$ & $36.52 \Omega$ \\
\hline
\end{tabular}

kept constant at 29 and $6 \mu \mathrm{m}$, respectively. The optimization results are shown in Table I. The optimizer has evidently tried to reduce electrode loss by increasing electrode thickness to its maximum value. Because the gap and width are constant, the only factor that influences inherent $V_{\pi}$ is buffer-layer thickness. It can be observed that the optimizer has chosen a very thin buffer layer to obtain lowest possible $V_{\pi}$ with given electrode gap and width.

This optimized modulator is compared with a resonantly enhanced modulator with a thick buffer layer $\left(T_{b}=1 \mu \mathrm{m}\right)$ to examine the trend on the choice of buffer-layer thickness. The loss increases by about $23 \%$ as the buffer-layer thickness decreased from 1 to $0.2 \mu \mathrm{m}$. This increase of electrode loss leads to a reduction of approximately $30 \%$ of normalized induced phase shift $\Delta \varphi$. However, inherent $V_{\pi}$ is reduced significantly from $17.4 \mathrm{Vcm}$ with $T_{b}=1 \mu \mathrm{m}$ to $11 \mathrm{Vcm}$ with $T_{b}=0.2 \mu \mathrm{m}$; then, the corresponding link gain is $1.7 \mathrm{~dB}$ better than the thick buffer-layer modulator.

The link-gain factor $M_{G}$ of the optimized RE-MZM is $12.5 \mathrm{~dB}$, which is a $5-\mathrm{dB}$ improvement over the traveling-wave modulator with the same electrode structure without terminations. Since two modulators with identical electrode structures are compared, their inherent $V_{\pi}$ are the same. Thus, a 5-dB link-gain improvement is only due to modulation efficiency improvement related to resonant enhancement.

\section{B. Optimization With Electrode Gap}

As investigated previously, the electrode gap has a great impact on both electrode loss and inherent $V_{\pi}$, but the net effect on link gain is not clear. The intention here is to use the optimization algorithm to identify the effect of electrode gap on the total link gain and optimization trends on the choice of electrode structure. Optimizations (Cases 2-16) were run for fixed electrode gaps ranging from 5 to $47 \mu \mathrm{m}$ in steps of $3 \mu \mathrm{m}$. All other parameters were set as variables.

The optimization results are summarized in Table II. There are several trends that can be clearly identified. The electrodes have become very thick to reduce electrode loss. The optimizer automatically seeks reduced inherent $V_{\pi}$ with a thin buffer layer. It can be seen from Table II that as electrode widths are variable, hot-electrode width was increased so that optical waveguides could be placed at optimal positions to get lowest inherent $V_{\pi}$. This phenomenon is clear when comparing the optimization results in Case 1 when hot-electrode width $W=6 \mu \mathrm{m}$ and the 
TABLE II

OPTIMIZATION RESULTS: CASES 2-16

\begin{tabular}{c|c|c|c|c|c|c|c|c}
\hline $\begin{array}{c}\text { Electrode } \\
\text { gap } \\
G(\mu \mathrm{m})\end{array}$ & $\begin{array}{c}\text { Electrode } \\
\text { width } \\
W(\mu \mathrm{m})\end{array}$ & $\begin{array}{c}\text { Buffer layer } \\
\text { thickness } \\
T_{b}(\mu \mathrm{m})\end{array}$ & $\begin{array}{c}\text { Electrode } \\
\text { thickness } \\
T_{e}(\mu \mathrm{m})\end{array}$ & $\begin{array}{c}\text { Electrode } \\
\text { length } \\
l(\mathrm{~cm})\end{array}$ & $\begin{array}{c}\text { Input } \\
\text { termination } \\
L_{1}(\mathrm{nH})\end{array}$ & $\begin{array}{c}\text { Output } \\
\text { termination } \\
L_{2}(\mathrm{nH})\end{array}$ & $\begin{array}{c}\text { Electrode } \\
\text { impedance } \\
Z_{0}(\Omega)\end{array}$ & $\begin{array}{c}\text { Microwave } \\
\text { index } \\
N_{m}\end{array}$ \\
\hline \hline 5 & 16 & 0.2 & 10 & 4.33933 & 5.06856 & 1.51647 & 18.7806 & 2.64174 \\
\hline 8 & 14 & 0.2 & 25 & 5.31395 & 2.82247 & 1.29947 & 17.6474 & 2.29318 \\
\hline 11 & 14 & 0.2 & 30 & 5.42172 & 2.25880 & 1.27669 & 19.4206 & 2.34486 \\
\hline 14 & 14 & 0.2 & 30 & 5.28503 & 2.10128 & 1.32216 & 21.9941 & 2.47059 \\
\hline 17 & 14 & 0.2 & 30 & 5.17885 & 1.99036 & 1.36745 & 24.1844 & 2.57001 \\
\hline 20 & 16 & 0.2 & 30 & 5.02233 & 1.85249 & 1.36391 & 25.4053 & 2.68918 \\
\hline 23 & 16 & 0.2 & 30 & 4.94903 & 1.78805 & 1.40112 & 27.0332 & 2.76017 \\
\hline 26 & 16 & 0.2 & 30 & 4.89082 & 1.73306 & 1.44306 & 28.5379 & 2.81834 \\
\hline 29 & 16 & 0.2 & 30 & 4.83459 & 1.69081 & 1.49429 & 29.8917 & 2.86993 \\
\hline 32 & 16 & 0.2 & 30 & 4.80724 & 1.67248 & 1.55486 & 31.5696 & 2.90463 \\
\hline 35 & 16 & 0.2 & 30 & 4.77277 & 1.64443 & 1.58582 & 32.8233 & 2.94226 \\
\hline 38 & 16 & 0.2 & 30 & 4.73732 & 1.61722 & 1.63740 & 34.0188 & 2.97636 \\
\hline 41 & 16 & 0.2 & 30 & 4.70796 & 1.59771 & 1.66494 & 35.1166 & 3.00755 \\
\hline 44 & 16 & 0.2 & 30 & 4.67935 & 1.57723 & 1.70789 & 36.1630 & 3.03548 \\
\hline 47 & 16 & 0.2 & 30 & 4.65543 & 1.55874 & 1.74487 & 37.1725 & 3.06037 \\
\hline
\end{tabular}

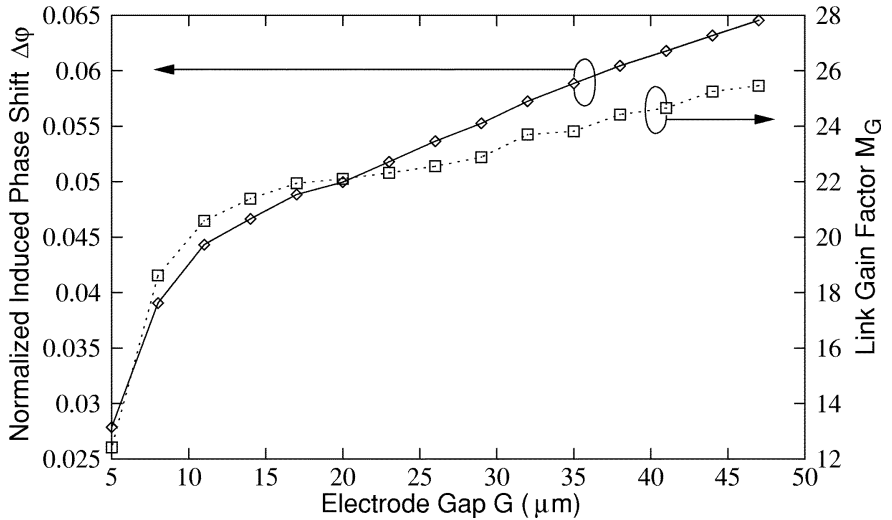

Fig. 8. Normalized induced phase shift $\Delta \varphi$ and link-gain factor $M_{G}$ of optimized resonantly enhanced modulators at $1.8 \mathrm{GHz}$.

case when the width was a variable with the same electrode gap of $29 \mu \mathrm{m}$. When the hot electrode is narrow, inherent $V_{\pi}$ is 11 $\mathrm{V} \mathrm{cm}$; when the hot electrode is wider to $16 \mu \mathrm{m}$, inherent $V_{\pi}$ is reduced to $8.2 \mathrm{Vcm}$. The net result is that the link-gain factor increases from 12.5 to $13.5 \mathrm{~dB}$ with a wider hot electrode.

Fig. 8 shows the relationship between the normalized phase shift $\Delta \varphi$ and the electrode gap $G$. When the gap is large, electrode attenuation drops (see Fig. 3). As a consequence, a strong standing wave is built in the electrode, which causes increased modulation depth. Modulation efficiency increases quite linearly with electrode gap when the gap is larger than $11 \mu \mathrm{m}$. However, the high inherent $V_{\pi}$ associated with this large electrode gap limits the link gain. Fig. 8 also presents the effect of electrode gap on link gain of resonantly enhanced modulators. It can be observed from Fig. 8 that although inherent $V_{\pi}$ is degraded with increasing gap, it is still possible to achieve a better link gain with large gap due to low loss. Thus, we can increase the gap at the expense of inherent $V_{\pi}$ to get strong modulation efficiency in order to improve link gain. Because electrode loss is frequency dependent, at higher operating frequency $(10 \mathrm{GHz}$,

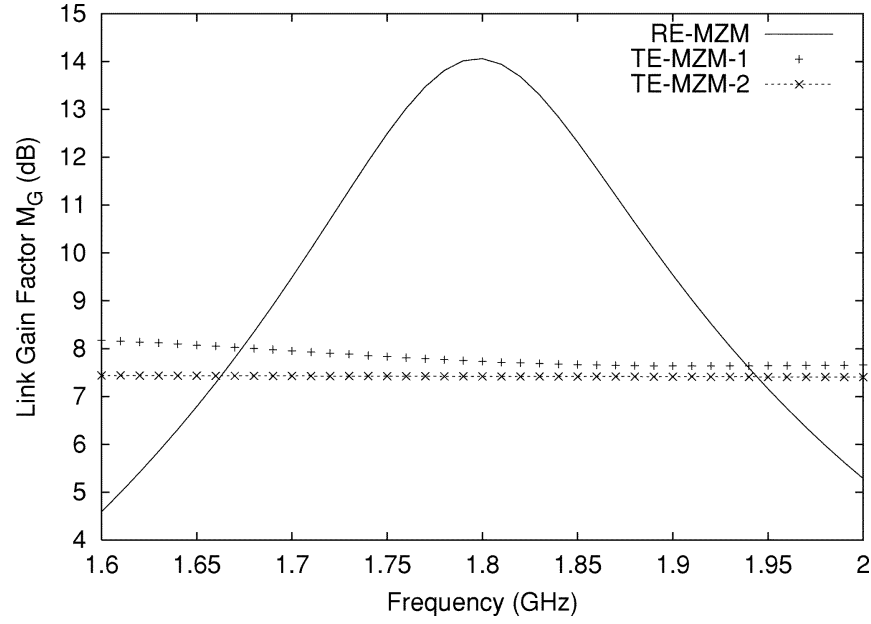

Fig. 9. Link-gain factor $M_{G}$ of the optimized resonantly enhanced modulator (RE-MZM) compared with conventional traveling-wave electrode modulator (TE-MZM-1) and two-step back-slot traveling-wave electrode modulator (TE-MZM-2).

for instance), if the gap becomes wider, electrode length can be increased in order to get longer interaction length to reduce drive voltage, as investigated in [11].

With a wider gap, the inductance of the input termination is also reduced. This is a desirable effect, because it will make the realization of inductive terminations with shorted CPW [22] easier.

\section{Link-Gain Comparison}

It is useful to identify the degree of improvement in terms of link gain that an optimized resonantly enhanced modulator offers over unenhanced traveling-wave electrode modulators. The link-gain factor of an optimized resonantly enhanced modulator with $47-\mu \mathrm{m}$ electrode gap and two unenhanced modulators are calculated from 1.6 to $2 \mathrm{GHz}$ and are shown graphically in Fig. 9. The first unenhanced modulator (TE-MZM-1) 


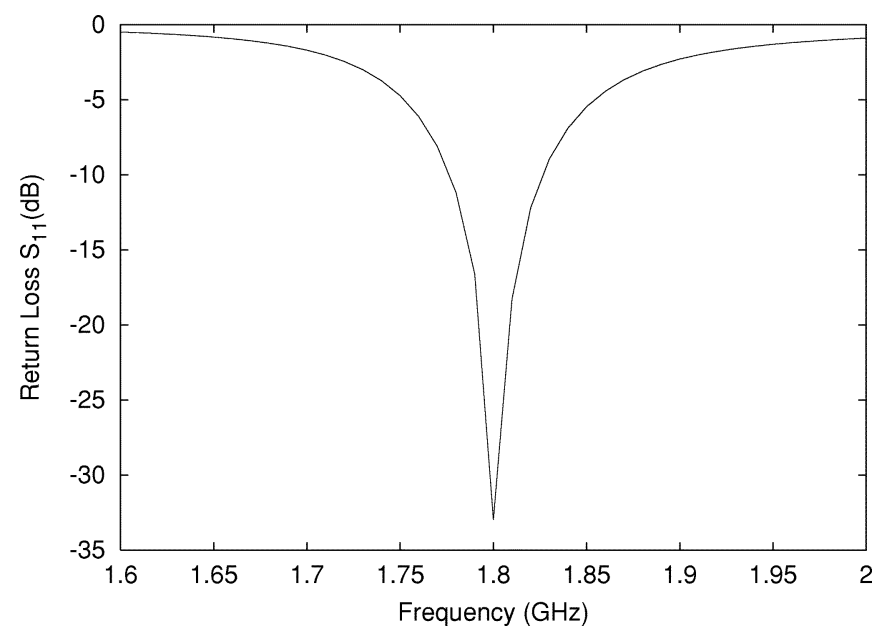

Fig. 10. Return loss of optimized resonant modulator. Electrode gap is $47 \mu \mathrm{m}$.

is the resonantly enhanced modulator with the inductive terminations removed. Instead, it is terminated with $50-\Omega$ load at the output port. The second unenhanced modulator (TE-MZM-2) is a high-performance broad-band modulator from the literature. This consists of a traveling-wave modulator on thinned $X$-cut $\mathrm{LiNbO}_{3}$ substrate with two-step back-slot structure [23]. At 1.8 $\mathrm{GHz}$, the optimized resonant-type modulator gives more than 6-dB improvement in link gain over both traveling-wave electrode modulators.

The RF return loss of the optimized resonant modulator with a 47- $\mu$ m electrode gap is shown in Fig. 10. It is clear that the resonantly enhanced modulator not only offers good link-gain enhancement but also provides excellent RF return loss (better than $-30 \mathrm{~dB}$ ) at the resonant frequency. Although the characteristic impedance of the electrode structure is fairly low $(37 \Omega)$, perfect impedance matching is still possible by optimizing the terminations.

\section{CONCLUSION}

In this paper, we have presented an investigation of resonantly enhanced optical modulators using a numerical optimization tool. FEM simulation, together with a numerical network model, has been employed to characterize the electrooptic response of resonant-type modulators. It is shown that electrode loss is the main factor that limits the modulation efficiency of resonantly enhanced modulators. By using thick electrodes and increasing the gap between hot electrode and ground plane to lower the attenuation, it is possible to obtain strong modulation efficiency with long electrodes. The high inherent $V_{\pi}$ associated with larger electrode gap can be compensated by using a thin buffer layer. For resonantly enhanced modulators fabricated on $X$-cut $\mathrm{LiNbO}_{3}$, hot electrodes can also be widened to optimal width to further reduce inherent $V_{\pi}$.

By optimizing electrode cross-sectional parameters, strong modulation efficiency is achievable with resonant-type electrodes, at the same time reducing inherent $V_{\pi}$. Strong modulation efficiency together with low inherent $V_{\pi}$ increases the link gain significantly, so that the modulators can be employed on radio-on-fiber links without the need of very high gain RF amplifiers at the remote stations.
The optimization tool and network model are not limited to the Fabry-Pérot type of resonantly enhanced modulators. They can be extended to investigate the performance of optical modulators with arbitrary electrode configurations such as short-path [9] double-stub [5] multiple-cavity resonant-type modulators as well as broad-band traveling-wave electrode and phase-reversal modulators.

A future investigation will attempt to practically realize the optimal configurations identified in this paper. It is anticipated, due to the resonant nature of the electrodes that the performance of the optimized resonantly enhanced modulators may be sensitive to fabrication tolerances. An investigation of this sensitivity and methods to minimize its impact will also be conducted as a future study.

\section{REFERENCES}

[1] E. Wooten, K. Kissa, A. Yi-Yan, E. Murphy, D. Lafaw, P. Hallemeier, D. Maack, D. Attanasio, D. Fritz, G. McBrien, and D. Bossi, "A review of lithium niobate modulators for fiber-optic communications systems," IEEE J. Select. Topics Quantum Electron., vol. 6, pp. 69-82, Jan.-Feb. 2000 .

[2] K. Noguchi, O. Mitomi, and H. Miyazawa, "Millimeter-wave Ti:LiNbO ${ }_{3}$ optical modulators," J. Lightwave Technol., vol. 16, pp. 615-619, Apr. 1998.

[3] A. Nirmalathas, D. Novak, C. Lim, R. Waterhouse, and D. Castleford, "Fiber networks for wireless applications," in Proc. 2000 IEEE 13th Annu. Meeting Conf. Lasers Electro-Optics Soc. (LEOS 2000), vol. 1, 2000, pp. 35-36.

[4] G. Gopalakrishnan and W. Burns, "Performance and modeling of resonantly enhanced $\mathrm{LiNbO}_{3}$ modulators for low-loss analog fiber-optic links," IEEE Trans. Microwave Theory Tech., vol. 42, pp. 2650-2656, Dec. 1994.

[5] T. Kawanishi, S. Oikawa, K. Higuma, Y. Matsuo, and M. Izutsu, "LiNbO ${ }_{3}$ resonant-type optical modulator with double-stub structure," Electron. Lett., vol. 37, no. 20, pp. 1277-1246, 2001.

[6] T. Kawanishi, Y. Matsuo, M. Izutsu, S. Oikawa, and K. Higuma, "Resonant-type optical modulator with double-stub structure," in Proc. IEEE 14th Annu. Meeting Lasers Electro-Optics Soc. 2001 (LEOS 2001), vol. 1, 2001, pp. 184-185.

[7] T. Kawanishi, S. Oikawa, K. Higuma, M. Sasaki, and M. Izutsu, "Design of $\mathrm{LiNbO}_{3}$ optical modulator with an asymmetric resonant structure," IEICE Trans. Electron., vol. E85-C, no. 1, pp. 150-155, 2002.

[8] T. Kawanishi, Y. Matsuo, M. Izutsu, S. Oikawa, and K. Higuma, "Reflection-type Mach-Zehnder $\mathrm{LiNbO}_{3}$ optical modulator with double-stub structure," in Proc. Conf. Lasers Electro-Optics Soc. Annu. Meeting, vol. 2, 2002, pp. 833-834.

[9] R. Krahenbuhl and M. Howerton, "Investigations on short-path-length high-speed optical modulators in $\mathrm{LiNbO}_{3}$ with resonant-type electrodes," J. Lightwave Technol., vol. 19, pp. 1287-1297, Sept. 2001.

[10] S. Oikawa, T. Kawanishi, K. Higuma, Y. Matsuo, and M. Izutsu, "Double-stub structure for resonant-type optical modulators using 20- $\mu$ m-thick electrode," IEEE Photon. Technol. Lett., vol. 15, pp. 221-223, Feb. 2003.

[11] M. Sugiyama, M. Doi, S. Taniguchi, T. Nakazawa, and H. Onaka, "Driver-less $40 \mathrm{~Gb} / \mathrm{s} \mathrm{LiNbO}_{3}$ modulator with sub-1 V drive voltage," in Tech. Dig. Conf. Optical Fiber Communication, vol. 70, 2002, pp. FB62-FB64

[12] Y. Visagathilagar, A. Mitchell, and R. Waterhouse, "Fabry-Pérot type resonantly enhanced Mach-Zehnder modulator," in Tech. Dig. Int. Topical Meeting Microwave Photonics (MWP'99), vol. 1, 1999, Cat. 99EX301, pp. 17-20.

[13] S. Hum, M. Okoniewski, and R. Davies, "Modulator structures for radio-on-fiber applications," in Proc. 14th Int. Conf. Microwaves, Radar Wireless Communications, vol. 1, 2002, pp. 37-40.

[14] A. Lindsay, G. Knight, and S. Winnall, "Photonic mixers for wide bandwidth RF receiver applications," IEEE Trans. Microwave Theory Tech., vol. 43, pp. 2311-2317, Sept. 1995.

[15] J. Jin, The Finite Element Method in Electromagnetics. New York: Wiley, 1993. 
[16] C. Kim and R. Ramaswamy, "Overlap integral factors in integrated optic modulators and switches," J. Lightwave Technol., vol. 7, pp. 1063-1070, July 1989.

[17] S.-J. Chang, C.-L. Tsai, Y.-B. Lin, J.-F. Liu, and W.-S. Wang, "Improved electrooptic modulator with ridge structure in $\mathrm{X}$-cut $\mathrm{LiNbO}_{3}$," J. Lightwave Technol., vol. 17, pp. 843-847, May 1999.

[18] A. Mitchell, M. Lech, R. B. Waterhouse, and D. M. Kokotoff, "Automatic search for efficient broadband microstrip patch antennas," in Proc. Asia-Pacific Microwave Conf. (APMC), vol. 3, 2001, pp. 1251-1254.

[19] L. Ingber, "Adaptive simulated annealing (ASA): Lessons learned," Control Cybern., vol. 25, no. 1, pp. 33-54, 1996

[20] L. Ingber and B. Rosen, "Genetic algorithms and very fast simulated reannealing: A comparison," Math. Comput. Model., vol. 16, no. 11, pp. 87-100, 1992

[21] Y. Visagathilagar, A. Mitchell, and M. Austin, "General theoretical model for resonantly enhanced optical modulators," in Proc. 8th OptoElectronics Communications Conf. (OEC), vol. 23, 2003, pp. 337-338.

[22] J. K. A. Everard and K. Cheng, "High performance direct coupled bandpass filters on coplanar waveguide," IEEE Trans. Microwave Theory Tech., vol. 41, pp. 1568-1572, Sept. 1993.

[23] J. Kondo, A. Kondo, K. Aoki, S. Takatsuji, O. Mitomi, M. Imaeda, Y Kozuka, and M. Minakata, "Low-drive-voltage $40 \mathrm{~Gb} / \mathrm{s}$ modulator on $\mathrm{X}$-cut $\mathrm{LiNbO}_{3}$ wafer," in Proc. 27th Eur. Conf. Optical Communication (ECOC '01), vol. 3, 2001, pp. 334-335.

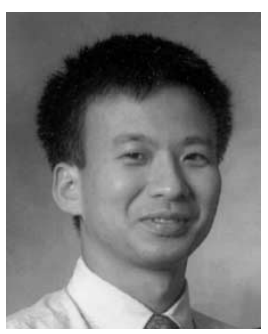

optical modulators.

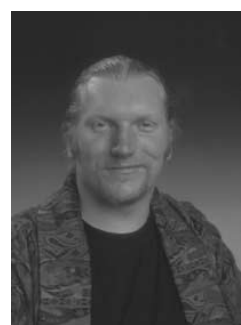

Arnan Mitchell (S'97-M'00) was born in Dublin, Ireland, on February 20, 1973. He received the B.Tech. degree (with honors) in optoelectronics from Macquarie University, Australia, in 1993, and the Ph.D. degree from the Royal Melbourne Institute of Technology (RMIT), Melbourne, Australia.

$\mathrm{He}$ is currently an Australian Photonics CRC Research Fellow at RMIT investigating broad-band and specialized integrated optical modulators and radiofrequency (RF) photonic components for communications and signal-processing applications. He maintains an active interest in the research of numerical methods required for the design of RF photonic integrated devices.

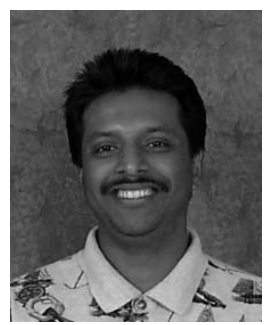

Yuvaraja S. Visagathilagar (S'93-M'02) was born in Colombo, Sri Lanka, on July 23, 1972. $\mathrm{He}$ received the B.Eng. degree in communications (with first-class honors) and the Ph.D. degree from the Royal Melbourne Institute of Technology (RMIT), Melbourne, Australia, in 1996 and 2003, respectively.

From 1999 to 2002, he was a Researcher undertaking the development of high-speed optical modulators as part of a defense contract. He is currently an Australian Photonics CRC Research Fellow with the School of Electrical and Computer Engineering, RMIT, where he investigates broad-band and specialized optical modulators, in particular narrow-band and resonantly enhanced modulators. His research interests are in theoretical and experimental investigation of microwave and microwave/photonic devices as well as integrating theses device with microwave devices/antennas for telecommunication and defense applications. 